\title{
Estimación de la talla en la evaluación nutricional de niños con parálisis cerebral
}

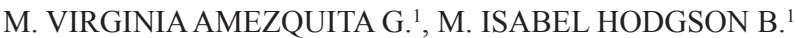 \\ 1. Médico Nutriólogo. Departamento de Gastroenterología y Nutrición Pediátrica, División de Pediatría, \\ Facultad de Medicina, Pontificia Universidad Católica de Chile.
}

\begin{abstract}
Alternatives to estimate stature during nutritional assessment of children with cerebral palsy

Introduction: The assessment of nutritional status plays a critical role in comprehensive child care, however, in children with cerebral palsy $(\mathrm{CP})$ is difficult to meet some reliable anthropometric measures, particularly height. Objective: To determine if the equations to estimate stature, developed and validated in CP populations of other countries, apply to our reality. Patients and Method: An anthropometric assessment in 60 children with cerebral palsy that included measurement of weight, height and body segments like tibia length (TL) and knee height $(\mathrm{KH})$ was performed. The height was estimated using the above described segments and the Stevenson et al. ${ }^{1}$ equations. The correlation and agreement between the measured and the estimated stature were evaluated. Results: Height could be reliably measured in 36 individuals and in all cases height was estimated. The correlation between the measured and the estimated lengths for TL and KH was 0.975 and 0.981 respectively $(\mathrm{p}<0.001)$. The analysis of agreement between the estimated and measured lengths showed on average a significant level of agreement, with an error of $-2.96 \mathrm{~cm}$ for TL and $0.21 \mathrm{~cm}$ for KH. Conclusions: The equations to estimate stature from the body segments, tibia length and knee height, are valid and useful to assess the linear growth in children with CP in our population.
\end{abstract}

(Key words: Cerebral palsy, nutritional assessment, height estimation, stature).

Rev Chil Pediatr 2014; 85 (1): 22-30

\section{RESUMEN}

Introducción: La evaluación del estado nutricional es fundamental en el cuidado integral del niño, sin embargo, en los niños con parálisis cerebral (PC) existe dificultad para obtener medidas antropométricas confiables, particularmente de la estatura. Objetivo: Determinar si las ecuaciones para estimar la talla, desarrolladas y validadas en población con PC de otras nacionalidades, tienen aplicabilidad en nuestro medio. Pacientes y

Recibido el 27 de mayo de 2013, devuelto para corregir el 22 de julio de 2013, segunda versión 15 de septiembre de 2013 , aceptado para publicación el 14 de octubre de 2013.

Este trabajo cumple con los requisitos sobre consentimiento /asentimiento informado, comité de ética, financiamiento, estudios animales y sobre la ausencia de conflictos de intereses según corresponda.

Correspondencia a:

M. Virginia Amezquita G.

E-mail: vickyamezquita4547@gmail.com 
Método: Se realizó una evaluación antropométrica en 60 niños con parálisis cerebral que incluyó la medición del peso, la talla y los segmentos corporales: Longitud de la tibia (LT) y altura de rodilla (AR). La talla se estimó usando los segmentos antes descritos y las ecuaciones de Stevenson et al ${ }^{1}$. Se evaluó la correlación y concordancia entre la talla medida y la estimada. Resultados: En 36 individuos la talla pudo ser medida de manera confiable y en todos los casos la talla también se estimó. La correlación entre la longitud medida y la estimada con LT y AR fue de 0,975 y de 0,981 respectivamente $(p<0,001)$. El análisis de la concordancia entre longitud estimada y longitud medida, en promedio, mostró un importante nivel de acuerdo, evidenciando un error sistemático de $-2,96 \mathrm{~cm}$ en la talla estimada con el segmento LT y de $0,21 \mathrm{~cm}$ con AR. Conclusiones: Las ecuaciones para estimar la talla a partir de los segmentos corporales, longitud de tibia y altura de rodilla, son válidas y útiles, para evaluar el crecimiento lineal de los niños con PC en nuestro medio, ante la dificultad de obtener medidas lineales convencionales.

(Palabras clave: Parálisis cerebral, evaluación nutricional, estimación de la talla, estatura).

Rev Chil Pediatr 2014; 85 (1): 22-30

\section{Introducción}

La Parálisis Cerebral es la causa más frecuente de discapacidad motora en la infancia ${ }^{2-4}$ y si bien, en nuestro país no existen estudios concluyentes al respecto, de acuerdo a las estadísticas de otros países y de las instituciones que se dedican al tratamiento de la enfermedad en Chile, se estima una incidencia de 2 a 3 por cada 1.000 recién nacidos vivos ${ }^{3,5-9}$.

Es conocido que las enfermedades crónicas tienen un fuerte impacto sobre el estado nutricional, y los niños con PC a menudo presentan problemas nutricionales, siendo los más frecuentes, la desnutrición, la falla de crecimiento lineal, la deficiencia de micronutrientes, la osteopenia y en menor proporción el sobrepeso $u$ obesidad $^{10,11}$.

La prevalencia de estas comorbilidades varía según las series publicadas. La desnutrición, la más frecuente, se ha documentado en rangos de $29 \%$ a $46 \%$ y su frecuencia aumenta con la edad, el mayor compromiso cognitivo y la severidad del daño neurológico ${ }^{12}$. El retraso de crecimiento lineal se ha reportado cercano al $23 \%$ y el exceso de peso en un $8 \%$ a $14 \%{ }^{13-20}$.

La desnutrición es especialmente prevalente en niños con formas graves de PC, donde la severidad del compromiso motor, el déficit cognitivo y las alteraciones en la motricidad facio-oro-linguo-deglutoria dificultan, de manera importante, la alimentación normal. Además, la incapacidad de comunicar sensaciones como el apetito o la saciedad, y la dificultad o imposibilidad de alimentarse por sí mismo, conducen a una mayor duración de las comidas, menor ingestión de alimentos o su rechazo y frecuentemente a vómitos ${ }^{21,22}$.

Por otro lado, la desnutrición afecta negativamente la calidad de vida de estos niños y se asocia a un mayor uso de servicios de salud, y a una menor participación en diversas actividades debido al deterioro de las funciones cerebrales, manifestado por irritabilidad o letargia y escasa motivación por el juego o la rehabilitación ${ }^{23,24}$.

La rehabilitación nutricional se asocia a una mejor salud general, mejor cicatrización de heridas y de las úlceras de decúbito, disminución de la espasticidad, de la irritabilidad y de la severidad del reflujo gastroesofágico ${ }^{25,26}$, así como, a una mejoría de la inmunidad, con disminución del riesgo de complicaciones $\operatorname{postoperatorias~}^{27,28}$. La literatura reporta, que el cuidado nutricional óptimo en los niños con deficiencias en el desarrollo neurológico, sería un factor significativo en la mejoría de su condición funcional y psicológica ${ }^{23-28}$.

La evaluación antropométrica en los niños con PC plantea dificultades especialmente en lo que se refiere a la obtención de medidas confiables de estatura. La presencia de contracturas articulares, la debilidad muscular, la escoliosis, los movimientos involuntarios y la poca cooperación del niño, hacen que la medición directa de la talla sea inexacta, poco confiable y a veces imposible de obtener ${ }^{25,29,30}$. Se ha reportado que la confiabilidad interobserva- 
dor, así como, los valores medios y las desviaciones estándar de las mediciones tomadas en posición de pie o en decúbito supino tendrían un valor similar en esta población ${ }^{31,32}$. Sin embargo, en los casos de PC grave, muchas veces tampoco es posible medir la longitud en decúbito supino ${ }^{1}$, por lo que es necesario considerar mediciones alternativas de los segmentos corporales, tales como la longitud de la tibia y la altura de rodilla, las que pueden compararse con los estándares disponibles en la literatu$\mathrm{ra}^{33-35}$. Estas han demostrado ser clínicamente útiles como medidas alternativas de la talla en niños y adolescentes con $\mathrm{PC}^{30-33,36}$, por su alta correlación con la talla en los niños $\operatorname{sanos}^{32-34} \mathrm{y}$ en los con $\mathrm{PC}^{1,30}$.

Además, usando estos segmentos y las ecuaciones publicadas provenientes de niños $\operatorname{sanos}^{32,34}$ o de niños con $\mathrm{PC}^{1}$ es posible estimar la talla. Stevenson et al. ${ }^{1}$, publicó ecuaciones obtenidas en una muestra de 172 niños con PC, demostrando una alta correlación entre la talla y los segmentos altura de rodilla, longitud de la tibia y longitud del brazo $(\mathrm{r}=0,98,0,97 \mathrm{y}$ $0,97$, respectivamente $p<0,05)$. El resultado del análisis multivariado en este estudio, mostró que las variables de edad, género o raza no influyen en las ecuaciones obtenidas.

A la fecha, en Chile no hay estudios publicados sobre la utilidad clínica del uso de segmentos corporales para estimar la talla en los niños con PC, aún cuando, la literatura científica internacional recomienda su uso ante la dificultad de obtener medidas lineales convencionales.

El objetivo de este estudio es determinar si las ecuaciones para estimar la talla, desarrolladas y validadas en población con PC de otras nacionalidades, tienen aplicabilidad en nuestro medio.

\section{Pacientes y Método}

Se reclutaron 60 niños con diagnóstico de $\mathrm{PC}$, con edades entre los 3 y 15 años, que tuviesen consentimiento informado y firmado por sus padres y/o cuidadores, y que acudiesen en forma regular a control a uno de los cuatro centros de rehabilitación de la Región Metropolitana: Instituto Nacional de Rehabilitación
Pedro Aguirre Cerda, Fundación Alter Ego, Instituto Teletón-Santiago y Centro de Rehabilitación Infantil "Luz y Esperanza" de la Fundación COANIL. Se excluyeron niños sin diagnóstico claro de PC o con otra condición concomitante con efecto confundente sobre el crecimiento (Ej., cardiopatía, genopatía, etc.).

La información se recopiló en el instrumento "Ficha de evaluación nutricional de niños con parálisis cerebral" diseñada para este estudio, incluyendo los antecedentes personales del paciente, el tipo y etiología de la PC y las variables antropométricas peso $(\mathrm{kg})$, altura en bipedestación o longitud en posición supina $(\mathrm{cm})$, longitud de la tibia $(\mathrm{cm})$ y altura de rodilla $(\mathrm{cm})$.

La altura de rodilla se define como la distancia comprendida entre la rodilla y el tobillo cada uno flectado en ángulo de $90^{\circ}$. La medición se realiza desde el talón a la superficie anterior del muslo sobre los cóndilos femorales. Para su determinación se uso el calibrador de Ross ${ }^{\circledR}$ (figura 1a). La longitud de la tibia se define como la distancia comprendida entre el borde supero medial de la tibia y el borde inferior del maléolo medial, con el niño sentado o acostado y una pierna cruzada horizontalmente sobre la otra. Para su determinación se uso una cinta métrica inextensible (figura 1b).

La estimación de talla se realizó con las ecuaciones publicadas por Stevenson et al ${ }^{1}$.

Longitud $(\mathrm{cm})=3,26 \times$ LT $(\mathrm{cm})+30,8$

Longitud $(\mathrm{cm})=2,69 \times \operatorname{AR}(\mathrm{cm})+24,2$

Utilizando la información del valor del coeficiente de correlación $r$, encontrada en este estudio, tanto para LT como AR, se calculó el número $\mathrm{n}$ del tamaño muestral para un nivel de seguridad del $95 \%$ y un poder estadístico del $80 \%$.

El material técnico usado fue una silla balanza mecánica marca Seca ${ }^{\circledR}$ (precisión de 50 g), balanza digital Seca ${ }^{\circledR}$ (precisión $10 \mathrm{~g}$ ), cinta métrica metálica flexible inextensible (precisión de $1 \mathrm{~mm}$ ) y caliper de Ross ${ }^{\circledR}$ (precisión de $1 \mathrm{~mm}$ ). El tallímetro fue diseñado especialmente para este trabajo y consistió en una tabla de medición supina de longitud máxima de 160 $\mathrm{cm}$ y una precisión de $1 \mathrm{~mm}$. Todas las medi- 

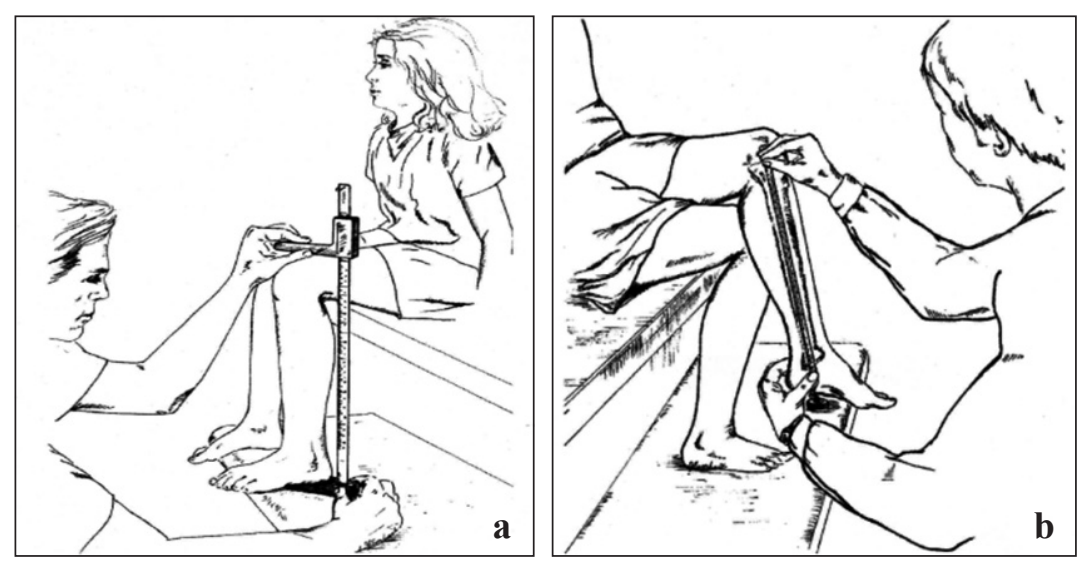

Figura 1. Medición de los segmentos corporales. a) Altura de rodilla; b) Longitud de tibia. ciones fueron realizadas por un único operador. Los segmentos LT y AR se midieron en dos oportunidades y el promedio de ambas se usó como valor final. En los casos de asimetría de extremidades o hemiplejia, las mediciones se realizaron en el hemicuerpo o segmento menos afectado, de lo contrario siempre se midió el lado derecho.

\section{Análisis estadístico}

Se usó el cálculo del tamaño muestral con la significancia del coeficiente de correlación de Pearson, para la medición de la intensidad de la relación lineal entre las variables talla medida y estimada, test de hipótesis de $\mathrm{r}$ basado en la distribución de Student para determinar si los coeficientes de correlación de Pearson eran estadísticamente significativos, y representación gráfica de la diferencia promedio de los dos procedimientos y su desviación estándar por el método de Bland Altman ${ }^{37}$. La confiabilidad de la medición de los segmentos se evaluó a través del cálculo del error técnico (ET) y del coeficiente de variación (CV). El análisis estadístico se realizó con el software Minitab. Para todo el análisis se estableció un nivel de significancia menor o igual a $0,05(\mathrm{p} \leq 0,05)$.

\section{Resultados}

Se reclutaron 60 niños, provenientes del Instituto de Rehabilitación Pedro Aguirre Cerda 48,3\% (n = 29), de la Fundación COANIL $21,7 \%$ ( $=13)$, del Instituto de Rehabilitación Teletón-Santiago $18,3 \%(n=11)$ y de la
Fundación Alter Ego 11,7\% (n=7). El 50\% presentaba una PC de tipo tetraparético, 33,3\% diparética, $5 \%$ hemiparética y en un caso la PC no estaba clasificada. Según el tipo de alteración motora, el 78,3\% tenía una PC espástica, el 20\% mixta, y en un caso era de tipo diskinética. El promedio de edad fue 8,2 $\pm 3,7$ años, $40 \%$ de sexo femenino, el peso promedio de $21,5 \pm 8,7 \mathrm{~kg}$, IMCz: $-0,74 \pm 1,9$ en niños $\geq$ de 6 años $(\mathrm{n}=38), \mathrm{P} / \mathrm{Tz}:-0,25 \pm 1,9$ en niños $<$ de 6 años $(\mathrm{n}=22), \mathrm{T} / \mathrm{Ez}:-2,2 \pm 1,6$, utilizando como referente las curvas de crecimiento de la Organización Mundial de la Salud 2007 (tabla 1$)$.

Tabla 1. Características generales de los 60 niños con parálisis cerebral. Distribución según tipo de trastorno y extensión del compromiso motor

\begin{tabular}{|ll|}
\hline Edad/años promedio $( \pm)$ DE & $8,2 \pm 3,7$ \\
Sexo $n(\%)$ & $H: 36(60)$ M: $24(40)$ \\
IMC ( score Z) & $-0,74 \pm 1,9$ \\
P/T (score Z) & $-0,25 \pm 1,9$ \\
T/E ( score Z) & $-2,2 \pm 1,6$ \\
Tipo de PC & $\mathbf{n}(\%)$ \\
Espástica & $47(78,3)$ \\
Mixta & $12(20,0)$ \\
Diskinética & $1(1,7)$ \\
Compromiso motor & $\mathbf{n}(\%)$ \\
Cuadriplejia & $30(50,0)$ \\
Diplejia & $20(33,3)$ \\
Hemiplejia & $9(15,0)$ \\
No tipificada & $1(1,7)$ \\
\hline
\end{tabular}



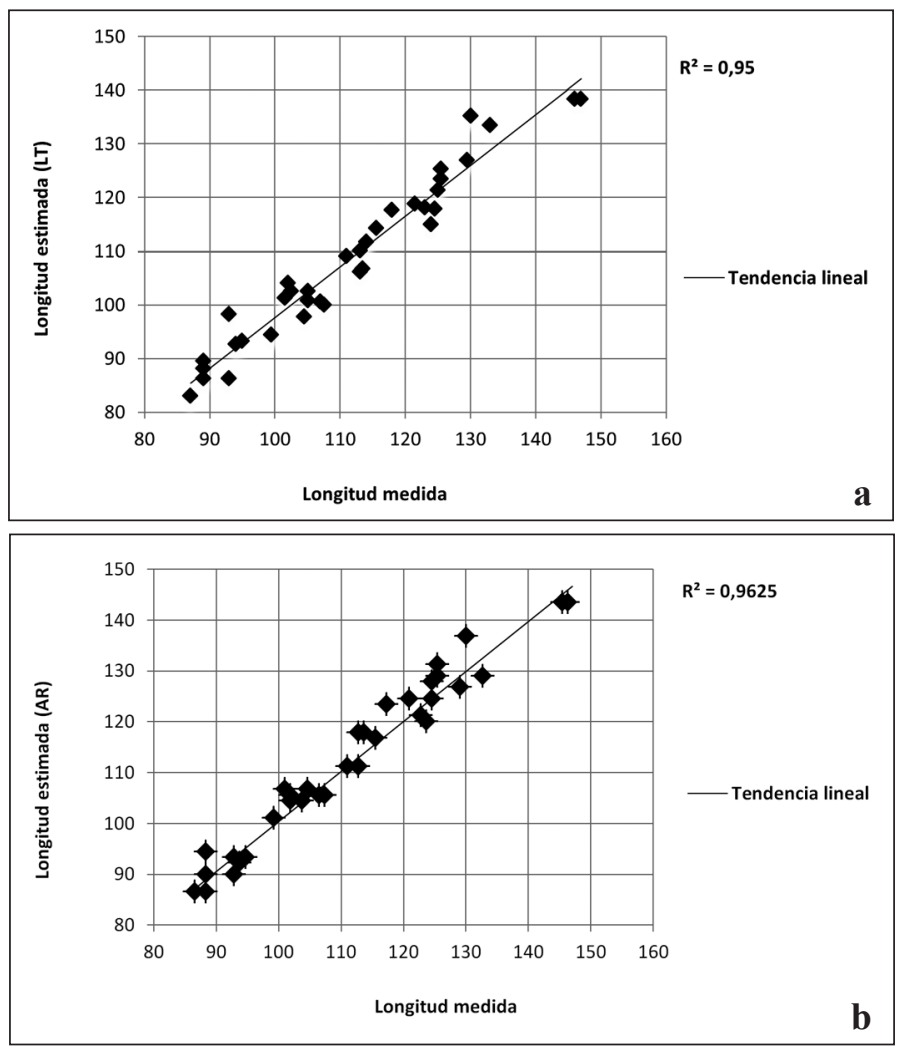

Figura 2. Correlación entre la longitud medida y la longitud estimada. a) Con segmento longitud de tibia. b) Con segmento altura de rodilla.

En 36 individuos (60\% de la muestra) fue posible medir la estatura en forma directa y en todos los casos este valor se obtuvo en posición decúbito supino. Ningún niño pudo ser medido en posición de bipedestación. En los 24 individuos restantes (40\%), la presencia de contracturas articulares, escoliosis, espasmos musculares o déficit cognitivo, impidió adosar los puntos de contacto de la cabeza, hombros, cadera y talones al plano del tallímetro, y obtener una medición confiable de la estatura. Del análisis del estudio, con estos 36 casos en que se logró medir la longitud, se obtuvo un coeficiente de correlación de Pearson entre la longitud medida y la estimada con LT de 0,975 (95\% intervalo de confidencia, 0,95 a 0,99 ) $\mathrm{r}^{2}=0,95$ y para AR de $0,981(95 \%$ de intervalo de confidencia, 0,96 a 0,99) $r^{2}=0,96$. Estos valores arrojaron un poder estadístico de $97 \%$ y $98 \%$ respectivamente (figuras 2 a y 2 b).

La longitud medida promedio de los 36 individuos fue de $111,6 \pm 15,8 \mathrm{~cm}$ y la de los segmentos LT y AR fue $23,9 \pm 4,7 \mathrm{~cm}$ y $32,6 \pm$
$5,9 \mathrm{~cm}$ respectivamente. La talla estimada promedio con LT fue $108,6 \pm 15,4 \mathrm{~cm}$ y con AR $111,7 \pm 15,9 \mathrm{~cm}$.

El error técnico (ET) de medición intraobservador para LT y AR fue 0,13 y $0,08 \mathrm{~cm}$ respectivamente, el coeficiente de variación $(\mathrm{CV})$ de 0,51 para LT y 0,25 para AR (tabla 2 ).

El método de Bland Altman mostró que cuando se estimó la talla con el segmento LT, la media de las diferencias fue de $-2,96 \mathrm{~cm}$, lo que se interpreta como un error sistemático de subestimación, con un rango comprendido entre 5,3 y -9,1 cm (figura 3a). En el caso de $\mathrm{AR}$, la media de las diferencias fue $0,21 \mathrm{~cm}$, lo que evidencia un error sistemático de sobreestimación, en un rango comprendido entre 6,1 y $-5 \mathrm{~cm}$ (figura $3 \mathrm{~b}$ ).

\section{Discusión}

En el grupo de niños con PC de nuestro estudio, la dificultad para obtener medidas confiables de talla alcanzó al 40\%, algo menor que lo 
Tabla 2. Confiabilidad de las mediciones de los segmentos. Error intraobservador de las mediciones obtenidas en el estudio y el reportado en la literatura

\begin{tabular}{|c|c|c|c|c|}
\hline \multicolumn{5}{|c|}{ Error Intraobservador } \\
\hline \multirow{3}{*}{ Segmento } & \multirow{2}{*}{\multicolumn{2}{|c|}{$\begin{array}{c}\text { Literatura } \\
\text { n = } 307\end{array}$}} & \multirow{2}{*}{\multicolumn{2}{|c|}{$\begin{array}{l}\text { Estudio } \\
n=60\end{array}$}} \\
\hline & & & & \\
\hline & $\mathrm{ET}^{*}(\mathrm{~cm})$ & $\mathrm{CV}^{* *}(\%)$ & ET $(\mathbf{c m})$ & CV (\%) \\
\hline $\mathrm{LT}^{\mathrm{a}}$ & 0,28 & 1,04 & 0,13 & 0,51 \\
\hline$A R^{a}$ & 0,22 & 0,61 & 0,08 & 0,25 \\
\hline
\end{tabular}

*Error técnico de medición intraobservador: $\sqrt{\frac{\sum d^{2}}{2 n}}$ donde d indica la diferencia entre mediciones pareadas de $\mathrm{n}$ sujetos, expresada en centímetros realizadas por un mismo observador. ${ }^{* *}$ Coeficiente de variación: (TE/promedio de las mediciones tomadas) x100 expresada

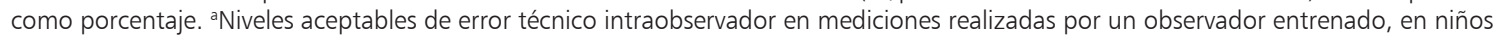
con $\mathrm{PC}^{37}$.
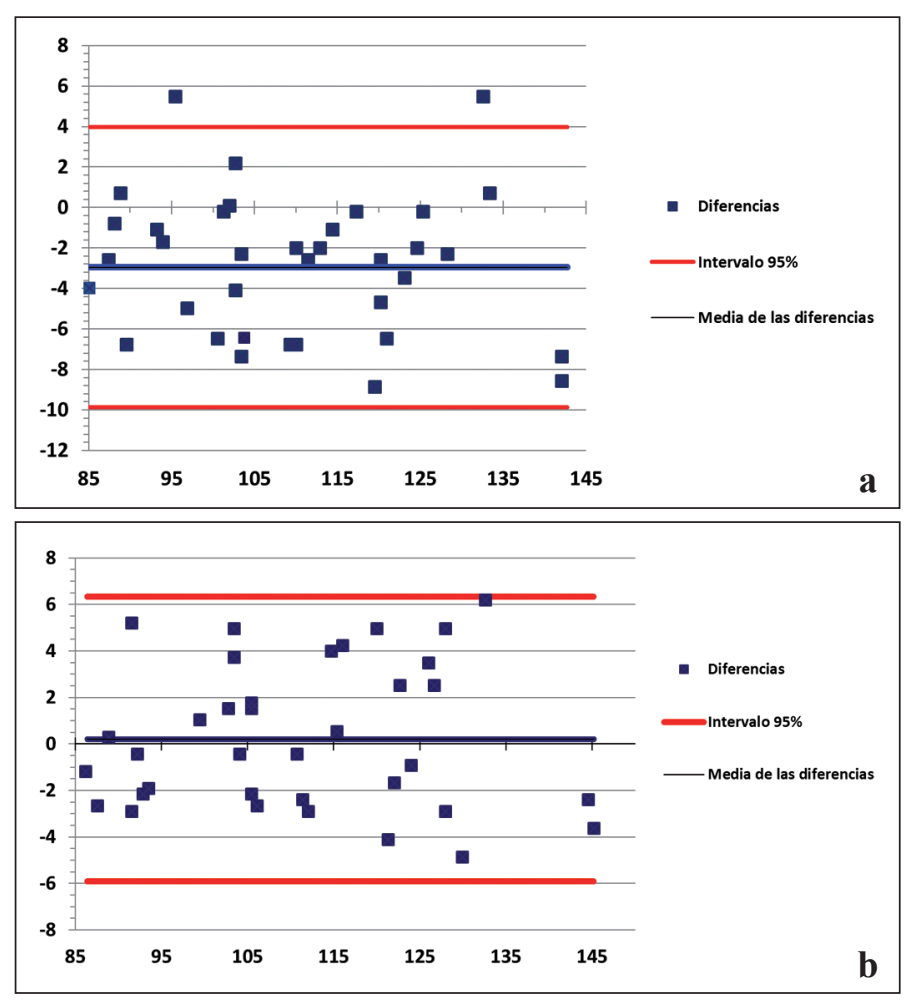

Figura 3. Concordancia entre la longitud medida y la longitud estimada. a) Con segmento longitud de tibia. b) Con segmento altura de rodilla.

reportado en otros estudios, $52 \%$ y $53 \%{ }^{1,33}$. En el $60 \%$ restante la talla se obtuvo en posición supina, ningún niño pudo ser medido en bipedestación como se recomienda sobre los 3 años de edad, debido a la alta proporción de niños con PC grave en esta muestra. Cabe destacar que, la medición de la longitud supina también es difícil de obtener en estos niños ${ }^{25,29}$, y considerando que en la práctica clínica habitual no se cuenta con un podómetro que permita medir niños sobre los $100 \mathrm{~cm}$ de longitud, resulta relevante realizar mediciones alternativas.

La edad promedio de los niños en que no fue posible medir la talla en forma directa fue mayor a la de los que pudieron ser medidos $9,5 \pm 3,4$ versus $7,4 \pm 3,7$ años, el compromiso motor también fue más severo en el grupo que no pudo ser medido, la prevalencia de PC tetraparética fue de $54,2 \%$ y $40 \%$ respectivamente. 
Los resultados obtenidos concuerdan con lo reportado en la literatura, que plantea que la dificultad para obtener medidas directas de talla ya sea en decúbito supino o bipedestación en los niños con PC, se incrementa con la edad y la severidad del compromiso motor ${ }^{1}$.

La correlación entre la longitud medida y la estimada por medición de los segmentos es similar a la descrita en la literatura ${ }^{1}$. Sin embargo, es importante destacar que la medición confiable de la altura de rodilla requiere de instrumental específico, calibrador de altura de rodilla, lo que limita la medición de este segmento en forma rutinaria. El segmento LT, en tanto, puede medirse con una cinta métrica flexible, y en manos de un operador entrenado permite obtener medidas precisas y confiables $^{33,38}$.

En nuestro estudio se obtuvo mayor concordancia entre la longitud medida y estimada cuando se uso el segmento AR que cuando se uso LT, probablemente, por el uso de un instrumento de mayor precisión para su medición. La concordancia obtenida con AR fue mejor a la descrita en la literatura, que reporta variaciones de hasta $12,7 \mathrm{~cm}$ por debajo a $11,8 \mathrm{~cm}$ por sobre la talla medida en niños con $\mathrm{PC}^{35}$.

El nivel de discrepancia entre la talla medida y la estimada por el método de Bland Altman tanto para AR como para LT es tolerable, considerando la gran dificultad para obtener mediciones directas de estatura en un alto porcentaje de niños con PC.

La confiabilidad de la medición de los segmentos LT y AR, mostró que el error técnico intraobservador de $0,13 \mathrm{~cm}$ y de $0,08 \mathrm{~cm}$ y el coeficiente de variación de 0,51 y 0,25 para LT y AR respectivamente, están dentro de los valores definidos como aceptables para asegurar la reproducibilidad ${ }^{35}$.

\section{Conclusiones}

Finalmente, podemos concluir de nuestro estudio que una alta proporción de niños no pudo medirse en forma convencional, o cuando se hizo su confiabilidad, fue dudosa. La longitud estimada con los segmentos AR y LT y las ecuaciones desarrolladas por Stevenson et $\mathrm{al}^{1}$, tienen una buena correlación, alta significancia estadística y buena concordancia, con la longitud medida en una muestra de niños chilenos con PC. Aunque estas ecuaciones fueron derivadas de niños con PC, deben usarse con precaución en niños con discapacidad motora severa ya que su validez no ha podido ser determinada, debido a que no es posible contar con medidas directas de longitud en este grupo específico. Por lo anterior, en estos niños podría ser más apropiado evaluar el crecimiento lineal usando curvas de segmentos corporales, evitando así potenciales errores en la predicción de la talla. No obstante, la estimación de la talla, constituye una alternativa útil en la práctica clínica para evaluar el crecimiento lineal de los niños con PC, cuando no pueden ser medidos en forma convencional y por ende, permite evaluar estado nutricional mediante el cálculo del índice de masa corporal (IMC).

\section{Agradecimientos}

A la Dra. María Loreto Ávila, pediatra del Instituto Nacional de Rehabilitación Pedro Aguirre Cerda (INRPAC), por su contribución a la recolección de datos y/o pacientes.

A los pacientes y sus familias, y a los funcionarios del Instituto Nacional de Rehabilitación Pedro Aguirre Cerda, la Fundación Alter Ego, el Instituto Teletón-Santiago y el Centro de Rehabilitación Infantil "Luz y Esperanza" de la Fundación COANIL, quienes con su colaboración y apoyo hicieron posible la realización de este estudio.

\section{Referencias}

1.- Stevenson RD: Use of segmental measures to estimate stature in children with cerebral palsy. Arch Pediatr Adolesc 1995; 149: 658-63.

2.- Siebes $R$, Wijnorks L, Vermeer A: Qualitative analysis of therapeutic motor intervention programmers for children with cerebral palsy: an update. Dev Med Child Neurol 2002; 44: 593-603.

3.- Reddihough DS, Collins KJ: The epidemiology and causes of cerebral palsy. Aust. J Physiotherapy 2003; 49: 7-12. 
4.- Koman LA, Paterson B, Shilt J: Cerebral Palsy. The Lancet 2004; 363: 1619-31.

5.- Bax M, Goldstein M, Rosenbaum P, Leviton A, Paneth $N$ : Proposed definition and classification of cerebral palsy. Dev Med Child Neurol 2005; 47: 571-6.

6.- Clark SL, Hankins GD: Temporal and demographic trends in cerebral palsy-fact and fiction. Am J Obstet Gynecol 2003; 18: 628-33.

7.- Winter S, Autry A, Boyle C, Yeargin-Allsopp M: Trends in the prevalence of cerebral palsy in a population based study. Pediatrics 2002; 110 (6): 1220-5.

8.- Nelson KB: The epidemiology of cerebral palsy in term infants. Ment. Retard Dev disabil Res Rev 2002; 8: 14650.

9.- Krigger $K W$ : Cerebral palsy: an overview. Am. Fam Physician 2006; 73: 91-100.

10.- Marchand V, Motil KJ: NASPGHAN Committee on Nutrition. Nutrition Support for Neurologically Impaired Children: A Clinical Report of the North American Society for Pediatric Gastroenterology, Hepatology, and Nutrition. JPGN 2006; 43: 123-35.

11.- Thomas AG, Akobeng AK: Technical aspects of feeding the disabled child. Curr Opin Clin Nutr Metab Care 2000; 3: 221-5.

12.- Stevenson RD, Hayes RP, Cater LV, Blackman JA: Clinical correlates of linear growth in children with cerebral palsy. Dev Med Child Neurol 1994; 36: 135-42.

13.- Stallings VA, Charney EB, Davies JC, Cronk CE: Nutritional status and growth of children with diplegic or hemiplegic cerebral palsy. Dev Med Child Neurol 1993; 35: 997-1006.

14.- Dahl M, Thommessen M, Rasmussen M, Selberg T: Feeding and nutritional characteristics in children with moderate or severe cerebral palsy. Acta Paediatr 1996; 85: 697-701.

15.- Troughton KE, Hill AE: Relation between objectively measured feeding competence and nutrition in children with cerebral palsy. Dev Med Child Neurol 2001; 43: 187-90.

16.- Sánchez-Lastres J, Eiris-Puñal J, Otero-Cepeda JL, Pavón-Belinchón P, Castro-Gago M: Nutritional status of mentally retarded children in north-west Spain. Anthropometric indicators. Acta Paediatr 2003; 92: 747-53.

17.- Shapiro BK, Green P, Krick J, Allen D, Capute AJ: Growth of severely impaired children: Neurological versus nutritional factors. Dev Child Neurol 1986; 28: 729-33.

18.- Krick J, Murphy-Miller P, Zeger S, Weight E: Pattern of growth in children with cerebral palsy. J Am Diet Assoc 1996; 96: 680-5.
19.- Stallings VA, Charney EB, Davies JC, Cronk CE: Nutrition-related growth failure of children with quadriplegic cerebral palsy. Dev Med Child Neurol 1993; 35: 126-38.

20.- Patrick J, Pencharz PB: Undernutrition in children with a neurodevelopmental disability. Canadian Medical Association Journal 1994; 151: 753-9.

21.- Srivastava VK, Laisram N, Srivastava RK: Cerebral Palsy. Indian Pediatr 1992; 29: 993-6.

22.- Reilly S, Skuse D, Poblete X: Prevalence of feeding problems and oral motor dysfunction in children with cerebral palsy: a community survey. J Pediatr 1996; 129: 877-82.

23.- Samson-Fang L, Fung E, Stallings VA, et al: Relationship of nutritional status to health and societal participation in children with cerebral palsy. J Pediatr 2002; 141: 637-43.

24.- Amundson JA, Sherbondy A, Van Dycke DC, Alexan$\operatorname{der} R$ : Early identification and treatment necessary to prevent malnutrition in children and adolescents with severe disabilities. J Am Diet Assoc 1994; 94: 880-3.

25.- Patrick J, Boland M, Stoski D, Murray GE: Rapid corection of wasting in children with cerebral palsy. Dev. Med Child Neurol 1986; 28: 734-9.

26.- Lewis D, Khoshoo V, Pencharz PB, Golladay ES: Impact of nutritional rehabilitation on gastroesophageal reflux in neurologically impaired children. Pediatr Surg 1994; 29: 167-9.

27.- Weber TR: A prospective analysis of factors influencing outcome after fundoplication. J Pediatr Surg 1995; 30: 1061-4.

28.- Jevsevar DS, Karlin LI: The relationship between preoperative nutritional status and complications after an operation for scoliosis in patients who have cerebral palsy. J Bone Joint Surg Am 1993; 75: 880-4.

29.- Tobis JS, Saturen P, Larios G, Posniak AO: Study of growth patterns in cerebral palsy. Arch Phys Med Rehabil 1961; 42: 475-81.

30.- Hogan SE: Knee Height as a Predictor of Recumbent Length for Individuals with Mobility-Impaired Cerebral Palsy. J Am Coll Nutr 1999; 18 (2): 201-5.

31.- Chumlea $W C$, Roche $A F$ : Nutritional anthropometric assessment of nonambulatory persons using recumbent techniques. Am J Phys Anthropol 1984; 63: 146-51.

32.- Chumlea WC, Guo SS, Steinbaugh ML: Prediction of stature from knee height for black and white adults and children with application to mobility-impaired or handicapped persons. J Am Diet Assoc 1994; 94: 1385-8. 
33.- Spender QW, Cronk CE, Charney EB, Stallings VA: Assessment of linear growth of children with cerebral palsy. Dev Med Child Neurol 1989; 31: 206-14.

34.- Gauld LM, Kappers J, Carlin JB, Robertson CF: Height prediction from ulna length. Dev Med Child Neurol 2004; 46: 475-80.

35.- Stevenson RD, Conaway M, Chumlea WC, et al: Growth and Health in Children with Moderate to Se- vere Cerebral Palsy. Pediatrics 2006; 118 (3): 1010-8.
36.- Bell KL, Davies PSW: Prediction of height from knee height in children with cerebral palsy and non-disabled children. Annals of Human Biology 2006; 33 (4): 493-9.

37.- Bland JM, Altman DG: Measurement error and correlation coefficients. BMJ 1996; 313: 41-2.

38.- Rogerson R, Gallagher M, Beebe A: Flexible tape is an appropriate tool for knee height measurement and stature estimation of adults with developmental disabilities. J Am Diet Assoc 2000; 100 (1): 105-7. 